\title{
The scalar does not decay at finite temperatures
}

\author{
Debasish Banerjee*, Rajiv Gavai and Sourendu Gupta \\ Department of Theoretical Physics, Tata Institute of Fundamental Research, \\ Homi Bhabha Road, Mumbai 400005, India \\ E-mail: \\ debasish@theory.tifr.res.in, gavai@tifr.res.in, sgupta@tifr.res.in
}

\begin{abstract}
We investigate medium effects on mesonic screening lengths for QCD with 2-flavours of dynamical staggered quarks on lattices with cutoff a=1/6T. Denoting the cross-over temperature by $T_{c}$, we vary the temperature $\mathrm{T}$ from $0.89 T_{c}$ to $1.92 T_{c}$, spanning both the hadronic and quark gluon plasma phases. While chiral symmetry restoration in the vector channel appears to take place near $T_{c}$, it is seen in the scalar channel only above $1.33 T_{c}$. Varying spatial lattice sizes, we find very little volume dependence in our results at $0.94 T_{c}$. We discuss the stability of the scalar meson at these temperatures.
\end{abstract}

The XXVIII International Symposium on Lattice Field Theory, Lattice2010

June 14-19, 2010

Villasimius, Italy

${ }^{*}$ Speaker. 


\section{Introduction}

When strongly interacting matter is heated, it is expected to go over to the Quark Gluon plasma (QGP) phase. Lattice Quantum chromodynamics (QCD) is extensively used to study the properties of matter across this transition. Even though the thermodynamic quantities and the equation of state is known in sufficient detail, more information about the plasma phase is needed to distinguish it from the hadronic phase.

The study of static correlation lengths constitute one such important clue. The low lying degrees of excitations of the plasma can be studied using the spatial correlation function of hadronic operators [1]. The screening mass is obtained from the exponential decay of the screening correlators at large spatial separations. Under certain analyticity assumptions, this screening mass can be related to the pole of the real time propagator of the corresponding operator as argued in [1]. These correlation functions, and the screening masses encode information about the interactions in the medium, indicating for example, whether certain symmetries broken at low temperatures are restored at high temperatures. It is known that at very high temperatures the screening masses of the mesons approach that of a free ideal gas, $2 \pi \mathrm{T}$ [2]. Moreover, finite volume corrections for the thermodynamic quantities can also be studied using the screening masses.

We investigate the spatial correlation functions for hadrons in 2-flavour QCD with staggered fermions in both the hadronic and the plasma phases. We study the pattern of chiral symmetry restoration across the cross-over in the screening spectrum. We study the finite volume effects on the correlation functions at low temperature and the possibility of the decay of the scalar meson.

\section{Technical Details}

The generation of the configurations used in the present study was reported in [3]. The lattices had a temporal extent of $N_{t}=6$ and spatial extent $N_{s}=24$, except in the finite volume study where spatial lattice sizes from 8 to 30 were used. Two flavours of light staggered quarks were used with the bare quark masses tuned such that the $T=0, m_{\pi} \approx 230 \mathrm{MeV}$ at all temperatures $\mathrm{T}$.

Point-to-point correlation functions for local meson operators in the pseudo-scalar (PS), scalar $(\mathrm{S})$, vector (V) and axial-vector (AV) channels were analyzed in detail. Denoting by $G(x, 0)$ the staggered propagator, the correlation functions projected to zero momentum are:

$$
C(z)=\frac{1}{N_{x}^{2} N_{t}} \sum_{x, y, t} \operatorname{Tr}\left[G(x, 0) G^{\dagger}(x, 0)\right] g_{S}(x)
$$

where $g_{S}(x)$ [4] are the staggered phase factors specific to the mesons in given quantum number channels. We have calculated only the connected part of the correlation functions, which correspond to the flavour non-singlet mesons. These correlation functions contain contributions from the respective parity partner as well, and hence are parameterized as

$$
C(z)=A_{1}\left(\mathrm{e}^{-m_{1} z}+\mathrm{e}^{-m_{1}\left(N_{z}-z\right)}\right)+(-1)^{z} A_{2}\left(\mathrm{e}^{-m_{2} z}+\mathrm{e}^{-m_{2}\left(N_{z}-z\right)}\right)
$$

Here $m_{1}$ and $m_{2}$ are the screening masses of the lightest natural parity meson appropriate for the operator used and its opposite parity partner. $A_{1}$ and $A_{2}$ are the respective normalizations. In this convention, the Goldstone pion is the non-oscillating part of the correlator and has positive $A_{1}$. 
The tolerance of the conjugate gradient (CG) algorithm was chosen to be $\varepsilon=10^{-5}$. This ensured that the systematic error in the meson correlation functions arising due to the matrix inversion was much lesser than the statistical errors.

The screening masses were extracted by fitting the correlation functions to the 2-mass fit form in eqn 2.2. As usual, the correlation between the different z-slices are incorporated through the covariance matrix and the fit values are obtained by minimizing the correlated- $\chi^{2}$.

\section{Results}

While our main results are on the temperature dependence of the screening mass spectrum on $N_{t}=6$ lattices, and the consequent study of the chiral symmetry restoration pattern as a function of temperature, we also present results for the spatial volume (in-)dependence of the screening masses of all the mesons near the critical end-point temperature.
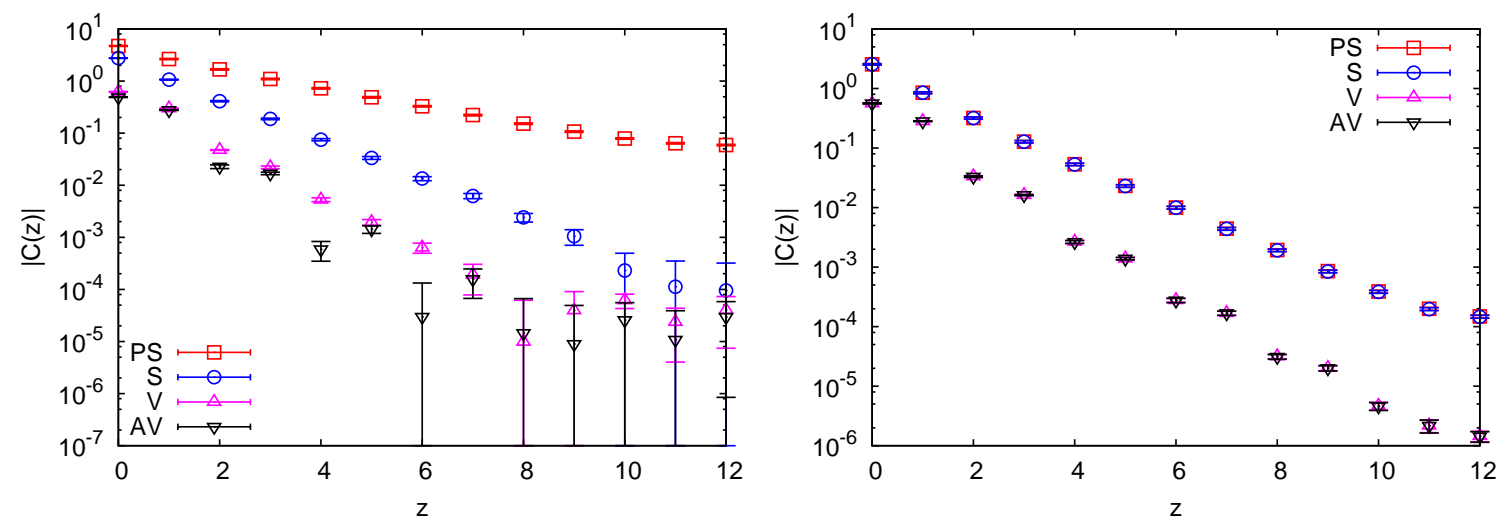

Figure 1: The correlators for all the mesons at $\mathrm{T}=1.92 T_{c}$ (left) and $\mathrm{T}=0.97 T_{c}$ (right). Note the prominent oscillations for the $\mathrm{V}-\mathrm{AV}$ mesons. We have plotted the absolute value of the correlators for the $\mathrm{V}$ and $\mathrm{AV}$ mesons.

\subsection{Screening spectrum}

We display our results for all the meson correlators in fig 1 at two different temperatures: 1.92 $T_{c}$ and $0.97 T_{c}$. The V/AV correlators show prominent oscillations at both temperatures. For the same statistics, there is more noise for the other mesons at large- $z$ and lower temperature than the pion, which is equally good at both temperatures. The pion correlation functions could be fit well to a single mass form at all temperatures, except the highest temperature (1.92 $\left.T_{c}\right)$ where we had to use the 2-mass fit. A full list of the screening masses will be given elsewhere[5].

To check the consistency of fitted masses, we also calculated the local masses. Due to the oscillating nature of the staggered fermions, the local masses were defined using correlators separated by $2 \mathrm{z}$-slices as done in [4]:

$$
\frac{C(z+1)}{C(z-1)}=\frac{\cosh \left[-m(z)\left(z+1-N_{z} / 2\right)\right]}{\cosh \left[-m(z)\left(z-1-N_{z} / 2\right)\right]} .
$$


The local masses so extracted are shown in Fig. 2 for the same two temperatures, $1.92 T_{c}$ and 0.97 $T_{c}$. The pion masses are the best determined in both the phases with small error bars and agree very well with the fitted values. Such an agreement are also found for the other mesons, although with larger bars.
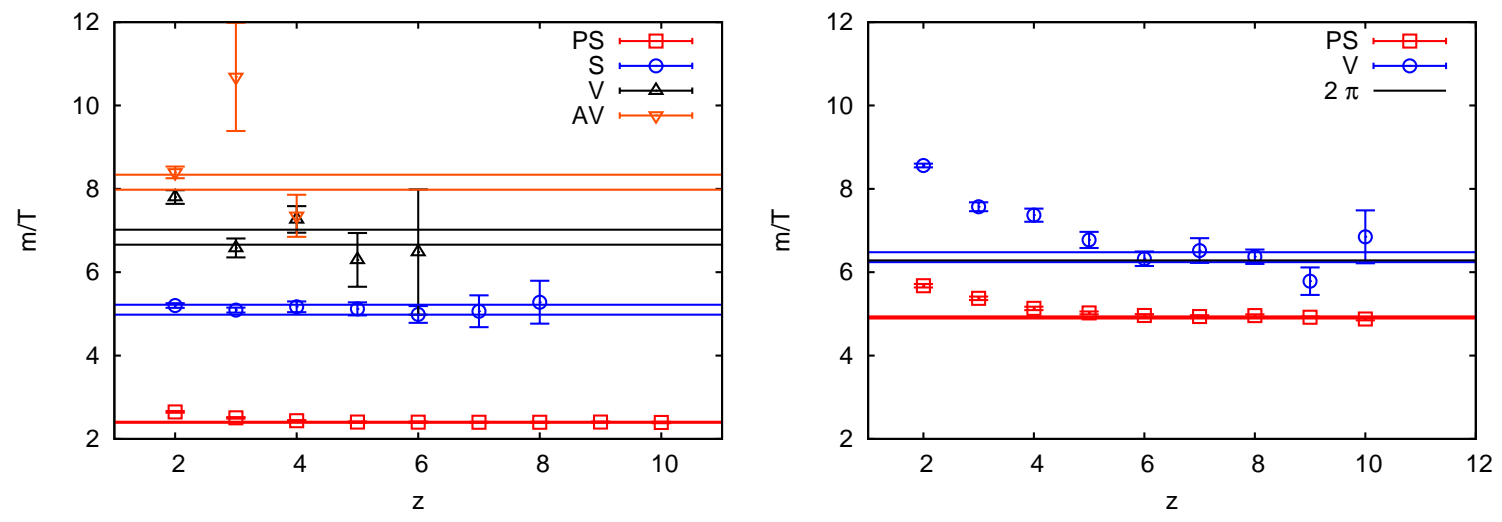

Figure 2: The extracted local masses for the mesons at $\mathrm{T}=0.97 T_{c}$ (left) and $\mathrm{T}=1.92 T_{c}$ (right). For $\mathrm{T}=1.92 T_{c}$, only the PS and V meson masses are shown since the S and AV exactly coincide with them. The black line coinciding with the lower blue line is the ideal gas limit.

The behaviour we discussed so far for both the hadronic and QGP phase is generic for other temperatures as well, with gradual changes in the fit parameters. Fig. 3 shows the fits to the PS and $\mathrm{V}$ correlators using the fitted values of the screening masses and corresponding amplitudes. The correlators have been scaled to depict the entire range in temperature on the same plot. The 2-mass nature of the fit used for the $\mathrm{V}$ correlators is very evident.

Finally, we plot the screening masses of the S, PS, V and AV mesons as a function of $T / T_{c}$ in Fig. 4. The chiral symmetry, broken at low temperatures, gets restored sufficiently away from the cross-over temperature $T_{c}$. While the PS and S mesons masses become degenerate only around $1.33 T_{c}$, the $\mathrm{V}$ and $\mathrm{AV}$ mesons are degenerate even at temperatures $\simeq T_{c}$. We note from Fig.2 that while the V/AV screening masses appear to be the same as the free theory continuum limit at about $2 T_{c}$, the screening masses of the PS/S mesons still differ from the ideal gas, and the V/AV mesons, by about $\sim 20 \%$. Our results confirm similar results obtained by other collaborations $[2,4,6]$.

\subsection{Finite Volume Study}

A finite volume study was done at $0.94 T_{c}$, since this is the phenomenologically interesting temperature where the QCD CEP is expected to lie at $\mu_{E}=1.6 T_{c}$ [3]. A range of lattices with $N_{s}=8,12,18,24$ and 30 were considered, where the smallest two had anisotropic spatial extents. From fig. 5, it is clear that the screening masses do not have significant volume dependence. All the lattices in our study are much bigger than the corresponding screening lengths of the mesons, which explains the absence of the finite volume effect associated with stable states.

Further, given that the mass of the scalar meson is more than twice the mass of the pion, we can discuss whether the decay of the scalar is possible. In the continuum, this decay is forbidden 

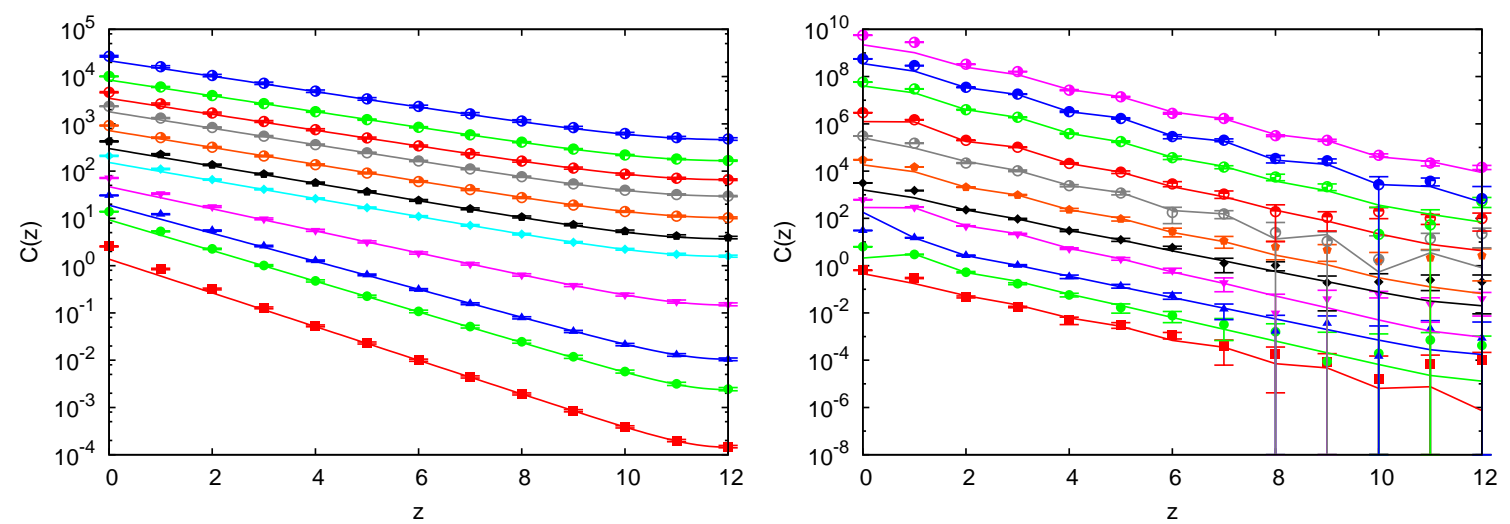

Figure 3: Single mass fits to the pseudo-scalar correlators (left) and the 2-mass fits to the vector correlators (right) for the entire temperature range studied here. Correlators have been appropriately scaled to make them fit in the same figure. The lines and symbols in each plot represent temperatures in units of $T_{c}$ in decreasing order from bottom: 1.92, 1.48, 1.33, 1.21, 1.01, 1.00, 0.99, 0.97, 0.94, 0.92, 0.89. (The lowest curve is for $1.92 T_{c}$ and the topmost is for $0.89 T_{c}$.)

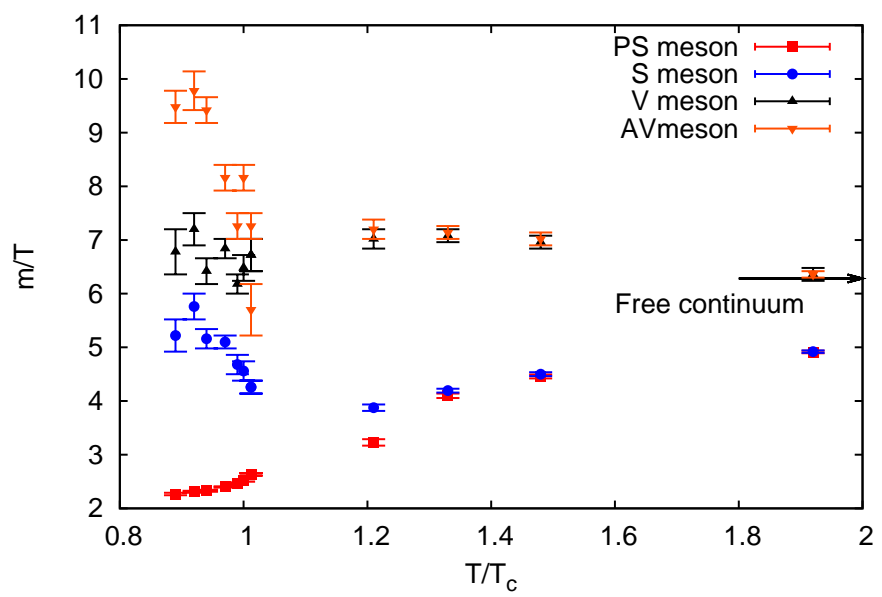

Figure 4: Masses of the PS, S, V and AV mesons a a function of $T / T_{c}$. The free continuum value of $2 \pi$ is indicated with an arrow.

by parity. On the lattice however, unphysical decays are known to occur [7]. In our case, parity and kinematical considerations imply that the scalar could only decay into the pion and one of its taste partners. A finite volume study could potentially answer this interesting question. Our results indicate that this decay does not occur. Fig 6 shows the scalar correlation functions are quite featureless as a function of volume. Further support comes from the fitted and the measured correlator normalization, $C_{S}(0)$, which are both independent of volume.

It is known that decays can be arrested if the volume is too small. In this context, we point out that we have worked with reasonably large volumes. The LT values in our study range from 1.33 


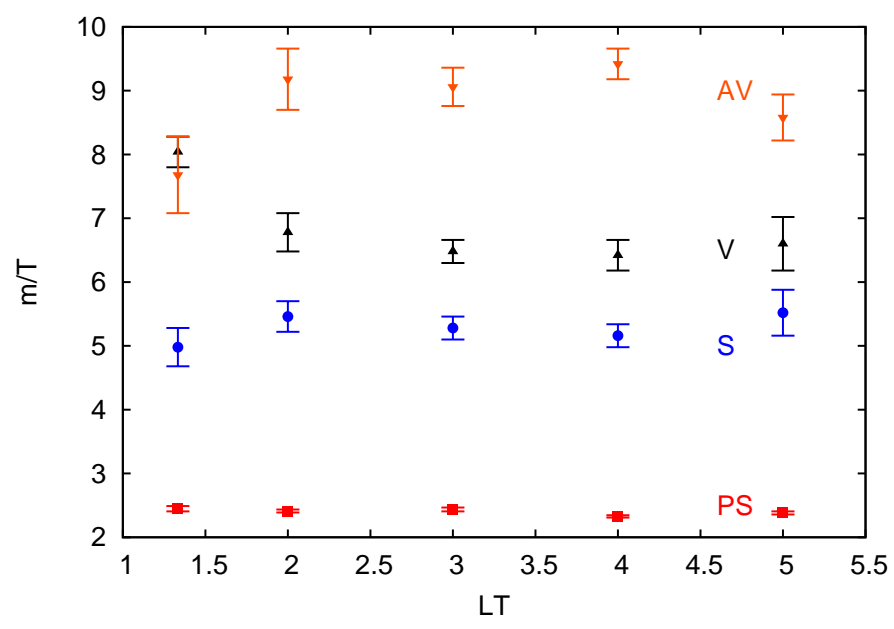

Figure 5: Masses of the PS, S, V and AV mesons a function of $L T$ at $T=0.94 T_{c}$.
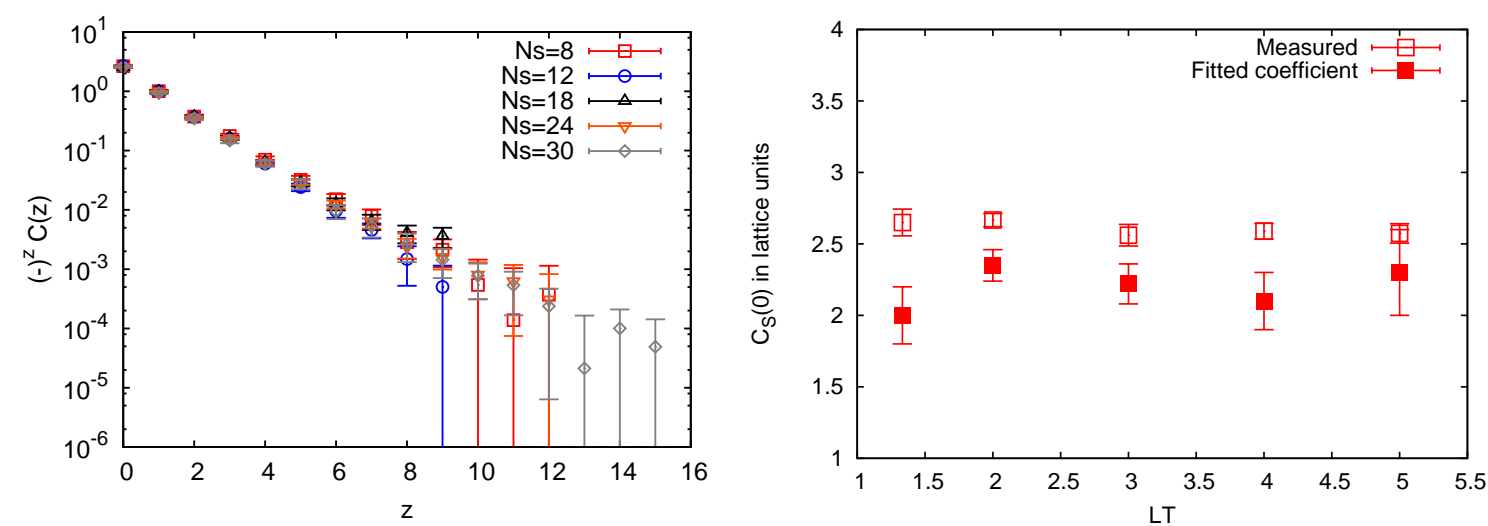

Figure 6: The upper figure shows the Scalar meson correlation functions. It is quite featureless as a function of the volume. The lower figure shows that both the measured and fitted value of $C_{S}(0)$ is independent of volume.

to 5 while $\mathrm{m}_{P S} \mathrm{~L}$ range from 2.4 to 12 .

\section{Summary}

We studied point-to-point correlation functions for mesons in the PS, S, V and the AV channels for two flavour QCD in the staggered fermion formulation. We extracted the corresponding screening masses and showed that while the screening masses of the V/AV mesons become degenerate by $T_{c}$, one needs to go up to $1.33 T_{c}$ to see the corresponding degeneracy of the PS/S mesons. Moreover, while by $2 T_{c}$ the V/AV screening masses are already seen to reach their continuum value, the PS/S screening masses are still 15-20\% away from the free theory value. Using a finite volume study, we find that the scalar correlation functions are insensitive to the change in spatial volume, 
which leads us to suspect that the scalar meson decay does not occur at finite lattice spacings at temperatures less than the cross-over temperature.

\section{Acknowledgments}

The computations were performed on the CRAY X1 of the Indian Lattice Gauge Theory Initiative (ILGTI) in TIFR, Mumbai. D.B. wishes to acknowledge useful discussions with Saumen Datta, Nilmani Mathur and Jyotirmoy Maiti.

\section{References}

[1] C. DeTar and J. B. Kogut Phys.Rev.D36(1987) 9

[2] K. Born et al (MTc Collaboration) Phys. Rev. Lett. 67, 302 (1991)

[3] R. V. Gavai, S. Gupta Phys.Rev.D78 (2008) 114503

[4] R. V. Gavai, S. Gupta and P. Majumdar Phys.Rev. D65 (2002) 054506

[5] D. Banerjee, R. V. Gavai and S. Gupta (in preparation)

[6] S. Mukherjee PoS LAT2007:210,2007

[7] C. Bernard et al Phys.Rev. D76 (2007) 094504 